\title{
NONSTANDARD ANALYSIS: ITS CREATOR AND PLACE
}

\author{
S. S. KUTATELADZE
}

On the 95th anniversary of the birth of Abraham Robinson

\begin{abstract}
This is a biographical sketch and tribute to Abraham Robinson on the 95th anniversary of his birth with a short discussion of the place of nonstandard analysis in the present-day mathematics.
\end{abstract}

This year, the world mathematical community recalls the memory of Abraham Robinson (1918-1974), an outstanding scientist whose contributions to delta-wing theory and model theory are the most convincing proofs of unity between pure and applied mathematics. Robinson created nonstandard analysis which is one of the most controversial, marvelous, and intriguing applications of logic to the core of mathematics.

\section{The Life's Signposts of Abraham Robinson}

Abraham Robinson was born on October 6, 1918 in Lower Silesia at a small Prussian town Waldenburg (today this is Watbrzych in Poland) In America Abraham was lately abbreviated as Abby.

Abraham, Abby, received the name in honor of his father who had died young not long before the birth of his younger son. The surname was written "Robinsohn" those days. Abby's father was a hebraist. talmudist, and zionist. Abby's grandfather on the mother's side was also a talmudist. Abby's uncle Isac was a famous and successful surgeon, and Abby together with his elder brother Saul spent summers at Isac's home near Vienna.

In 1925 Abby's mother, Lotte Robinsohn, moved with her two boys to Breslau, the capital of Silesia, where there was a large Jewish community. The brothers learned in a Jewish private school founded by Rabbi Max Simonson, who took great care of the junior Robinsohns,

Date: June 15, 2013.

${ }^{1} \mathrm{Cp}$. [1]-3 for biographical details.

${ }^{2} \mathrm{~A}$ hebraist is an expert in Hebrew Studies. 
remarking that "the big boy was an extremely gifted child, but the little one was a genius."

After Hitler seized power in 1933, Robinsohns emigrated to Palestine. The family settled in Jerusalem, where Abby went to the Rehavia Secondary School, appraised with the excellency of his Hebrew. He and his brother enlisted into Haganah, the illegal organization for defence against Arabs. In due time Haganah turned into a basis of the Army of Defense for Israel (Tzahal).

In 1936 Robinson entered the Einstein Mathematical Institute which was actually the Mathematics Department of the Hebrew University of Jerusalem. His mentor was Abraham Fraenkel.

In 1938 the first paper of Abby appeared in the Journal of Symbolic Logic (with $\mathrm{h}$ in the author's surname). In January of 1940 Robinson and his companion Jacob Fleischer moved to Paris where Abby enrolled in Sorbonne. But the Germans occupied Paris in June, and Abby together with Fleischer escaped to England through Bordeaux. In England he joined the Free French that was collected by de Gaulle. He became a sergeant of the Free French Air Force. Although Abby was a subject of the British Crown his German origins had hampered his entrance into the British Army for a time being. Miraculously enough, Abby's merits came into play. He had helped a familiar French Captain to make a memorandum on aircraft wings for the Ministry of Aircraft Production, and he was soon reassigned to the British Air Force and transferred to the Royal Aircraft Establishment in Farnborough as an assistant (grade 3) in the British Ministry of Aircraft Production.

In December of 1942 Robinson wrote to his supervisors in Jerusalem that he had decided to participate in the general struggle against Fascism and apply his knowledge in applied mathematics to this end. He remarked that there was no effort for him to turn to applied problems. Robinson addressed the problem of comparison between single-engine and twin-engine planes for which he suggested an analog of the variational method by Ludwig Prandl. He also worked on the problem of structural fatigue and collapse of a flying boat.

In 1944 Robinson married Renée Kopel, a fashion photographer. Abby lived with Renée up to his terminal day.

Robinson was a member of the group studying the German V-2 missiles as well as a mission of the British Intelligence Objectives Subcommission which concerned intelligence gathering about the aerodynamical research in Germany. In 1946 Robinson returned to Jerusalem to pass examinations for the Master degree. The results were as follows: "physics good, mathematics excellent." In this short period Abby worked together with Theodore Motzkin. 
In 1946 the Royal College of Aeronautics was founded in Cranfield near London. Robinson was offered the position of a Senior Lecturer with salary 700 pounds per year. It is worth mentioning that Robinson was the only member of the teaching staff who learned how to pilot a plane. In Cranfield Abby became a coauthor of delta-wing theory for supersonic flights, and in 1947 he learned Russian in order to read the Soviet scientific periodicals.

To gain the PhD degree, Robinson joined the Birkbeck College which was intended for mature working students and provided instructions mainly in the evening or on weekends. Abby's supervisor in the college was Paul Dienes, a Hungarian specialized mainly in function theory. Dienes instigated Abby's interest in summation methods (which resulted lately in Abby's work with Richard Cooke who also taught in the Birkbeck College). Dienes was a broad-minded scientist with interests in algebra and foundations. In 1938 he published the book Logic of Algebra, the topic was close to Abby's train of thoughts. In this background Robinson returned to logic and presented and maintained the PhD thesis "On the Metamathematics of Algebra" in 1947.

In 1951 Robinson moved to Canada where he worked at the Department of Applied Mathematics of Toronto University. He delivered lectures on differential equations, fluid mechanics, and aerodynamics. He also supervises postgraduate students in applied mathematics. Abby worked on similarity analysis and wrote "Foundations of Dimensional Analysis" which was published only after his death in 1974.

Robinson was the theorist of delta-wing, but his Farnborough research in the area was highly classified. In Toronto Robinson wrote his magna opus in aerodynamics, Wing Theory, which was based on the courses he delivered in Cranfield as well as on his research in Canada. Robinson invited as a coauthor John Laurmann, his former student in Cranfield. The book addressed airfoil design of wings under subsonic and supersonic speeds in steady and unsteady flow conditions. James Lighthill, the creator of aeroacoustics and one of the most prominent mechanists of the twentieth century, appraised most of the book as "an admirable compendium of the mathematical theories of the aerodynamics of airfoils and wings." Robinson performed some impressive studies of aircraft icing and waves in elastic media, but in the mid-1950s his interest in applied topics diminished had been fading. Robinson continued lecturing on applied mathematics, but arranged a seminar of logic for a small group of curious students.

In 1952 Robinson participated in the second Colloquium on Mathematical Logic in Paris. He made a memorisable comment on the about the "wings of logic." Louis Couturat stated that symbolic logic gave wings 
to mathematics, which involved the objection by Henry Poincaré that instead of giving it wings logic had only put mathematics in chains. Robinson rebuffed that however great the mathematician Poncaré may had been he was wrong about logic.

The Summer Institute in Logic held in Cornell was one of the most important events for Abby in 1957, when he had already sought for a job beyond Canada. Paul Halmos was the initiator of this gathering under the auspices of the American Mathematical Society. He wrote to Edward Hewitt who supervised summer institutes in the AMS that logic is a live subject developing rapidly without any support from "an admiral of the navy or a tycoon of industry." Leon Henkin and Alfred Tarski backed up Halmos's proposal. The meeting in Cornell marked the start of the rapid progress of logic in the USA. Robinson delivered three lectures on relative model completeness and elimination of quantifiers, on applications of field theory and on proving theorems "as done by man, logician, or machine." it is curious that Halmos had proclaimed himself to be a "logician humoris causa." Perhaps, his future invectives against nonstandard analysis demonstrate this status of his. $3^{3}$

In 1957 Robinson had left Canada and returned to his alma mater in Israel, where he delivered the compulsory courses on linear algebra and hydrodynamics and a special course on logic. In 1959 he was invited to read a course in fluid mechanics in the Weizmann Institute. Although Abby's contribution to applied mathematics was fully acknowledged, the place of his studies in the area had slowly faded out. But Robinson never lost his admiration of applications. Alec Young, a specialist in wing theory, remarked that everyone felt that "the applied mathematician in Abby was never far away," ready to meet any enticing challenge of praxis. After retirement of Fraenkel, Robinson became the dean of the Mathematics Department of the Hebrew University of Jerusalem.

In 1960 Robinson spent a sabbatical in Princeton. At the 1960 International Congress for Logic, Methodology, and Philosophy of Science he made the talk "Recent Developments in Model Theory" in which he gave a comprehensive survey of the pioneering works by Anatoly Maltsev, thus opening them in fact to the logicians of the USA. Robinson strongly emphasized the importance of the Maltsev studies demonstrating how the direct application of model theory led to particular algebraic results.

Soon Robinson was invited to make a plenary talk at the silver anniversary meeting of the Association for Symbolic Logic which took

\footnotetext{
${ }^{3}$ Cp. [4, pp. 202-206].
} 
place on January 24, 1961. This date has become the birthday of nonstandard analysis. In summer of 1961 Robinson had been invited to work in the University of California, Los Angeles (UCLA), where he moved in July, 1962. One of the first scholars who shared the ideas of nonstandard analysis was Wim Luxemburg, an outstanding specialist in functional analysis who primarily studied Banach lattice theory In may of 1962 Robinson wrote to Luxemburg: "For some time now I have been thinking about problems in Functional Analysis but so far as I can see our activities also may intersect there. Altogether, so far as my standard duties permit, I am now living in a non-standard mathematical world...." That was the manner he lived up to the end of life.

Robinson tried to demonstrate to richness of the new ideas in most diverse problems. He wrote on the technique of nonstandard analysis in theoretical physics, studied nonstandard points on algebraic curves, developed applications of the tools to large exchange economies, to integration of differential equations, to summations methods, and so on $\sqrt[5]{5}$

In the 1960s Robinson ranked as one of the most popular figures of the mathematical community. In 196 he was in the center of attention of the participants of the first international conference on nonstandard analysis which was arranged by Luxemburg in Caltech. In 1967 Robinson's book [8] was translated into Russian. But his magna opus on nonstandard analysis was never published in Russian partly in view of the rise of antisemitism in the academic community of the USSR in those years.

In 1968 Robinson was invited by Nathan Jacobson to leave UCLA for Yale, where Abby became a tutor of a large group of young logicians. In 1970. Abby made an invited plenary talk at the International Congress of Mathematicians in Nice on "Forcing in Model Theory." In 1971 he received Sterling Professorship, delivered a Hedrick lecture at the summer meeting of the Mathematical Association of America, made a talk at the Fourth International Congress for Logic, Methodology, and Philosophy of Science in Bucharest, etc. In 1972 Abby was elected to the American Academy of Arts and Science, and in 1973 the Dutch Mathematical Society decorated Abby with the Brouwer Medal.

The contributions by Robinson were highly appraised by the logic genius of the twentieth century Kurt Gödel who saw Robinson as his

\footnotetext{
${ }^{4} \mathrm{Cp} .[5],[6]$.

${ }^{5} \mathrm{Cp} .[7]$ - 11 .
} 
successor in Princeton. Gödel wrote: "there are good reasons to believe that nonstandard analysis, in some version or other, will be the analysis of the future", remarking that "his theory of infinitesimals and its application for the solution of analytical problems seems to me of greatest importance." 6 Unfortunately, Robinson could not move to Princeton. In November of 1973 Abby had begun to fell strong stomach pains, and the doctors found a nonoperable cancer of the pancreas. Robinson passed away on April 11, 1974 at the age of 55.

\section{Models of Nonstandard Universes}

Many incomprehensible and obfuscating words can be said about the nonstandard models of set theory and the methods of analysis which are based on them. Some other possibilities are open that we will pursue.

From antiquity mathematics bases on points and numbers. The most ancient and important method of research consists in representing numbers by points. This is the simplest example of modeling for study the properties of some objects (numbers) by the others - depicting numbers by points.

Let us elaborate this example by recalling the definitions of points and numbers which are given by Euclid in his Elements. By Definition I of Book VII a monad is "that by virtue of which each of the things that exist is called one." Euclid proceeds with Definition II: "A number is a multitude composed of monads." Note that the presentday translations of the Euclid treatise substitute "unit" for "monad."

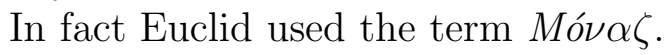

If we look at these definitions from the modern set-theoretic positions we can assert that two naturals are a pair of sets one of which includes the other. But two distinct points has no common elements and so are disjoint as sets. The points of Euclid are the predecessors of the modern representatives of the empty set which are called "atoms."

So, the presentation of numbers by points on the straight lines does not preserve the relations of modeled objects as sets - intersections are not retained. In other words, the membership between sets is not modeled properly.

A set theoretic model is called nonstandard, if the images of modeled objects fail to preserve membership. This definition belongs to Leon Henkin. Therefore, the common method of depicting numbers by points is an example of nonstandard set theoretic modeling.

\footnotetext{
${ }^{6} \mathrm{Cp} .[12]$.
} 
Let us look at this angle on some classical examples of modeling in mathematics:

(a) The Poincaré model of non-Euclidean geometry preserves intersections of straight lines and is standard in this respect.

(b) The classical presentation of a separable Hilbert space as the $l_{2}$ sequence space and the $L^{2}(-\pi, \pi)$ space of square-integrable functions gave an example of nonstandard modeling. Indeed, the functions sin and cos intersect as sets, and the abscissas of the intersection points are interesting. The presentations of this functions in $l_{2}$ has an uninteresting intersection.

Therefore, the Riesz-Fisher Isomorphism Theorem for Hilbert spaces of the same Hilbert dimension uses in fact a nonstandard model, which surely does not diminish its value and beauty.

Thus example reveals the main particularity of nonstandard modeling. Nonstandard models are very sensible in regard to what and how we model and verify claims. In other words, verification, i. e. discrimination between true and false propositions, must be explicitly defined.

The above demonstrates that the nonstandard methods of analysis, i. e., the techniques based on simultaneous consideration of standard and nonstandard set-theoretic models, is not something especial, indecent, or brand-new, We speak prose but the statement of the fact does make literature critics blush. We may say that today the possibilities of nonstandard modeling are elaborated much more than we did it before.

Return to the most popular and important nonstandard method of analysis - representation of numbers by points. Technically speaking, we depict a number $a$ by the singleton $\{a\}$. Clearly, the numbers, say 4 and 5, intersect, whereas their images $\{4\}$ and $\{5\}$ are disjoint.

Proceed by analogy with all sets, the elements of von Neumann universe $\mathbb{V}$ :

$$
*: a \in \mathbb{V} \rightsquigarrow A:=\{a\} \in \mathbb{V} \text {. }
$$

As usual,

$$
\mathbb{V}:=\bigcup_{\alpha \in \mathrm{On}} V_{\alpha}
$$

where

$$
V_{\alpha}:=\bigcup_{\beta \in \alpha} \mathscr{P}\left(V_{\beta}\right)
$$

i. e., $V_{\alpha}=\left\{x \mid(\exists \beta)\left(\beta \in \alpha \wedge x \subset V_{\beta}\right)\right\}$. 


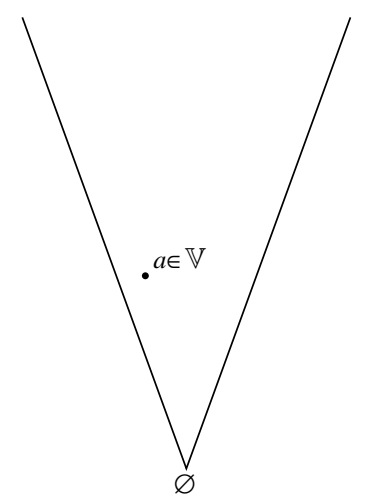

Von Neumann universe

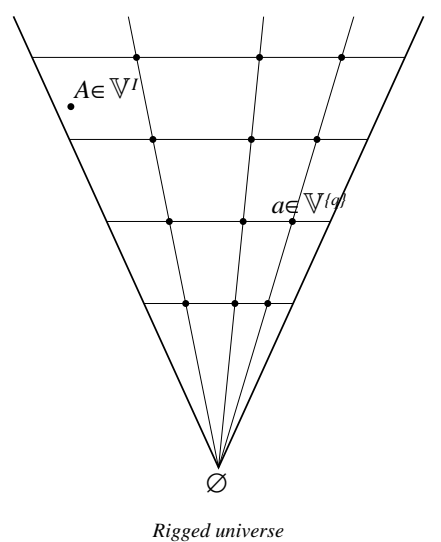

Rigged universe

There is another way of viewing the construction. Consider

$$
\mathbb{V}_{q}:=\{(q, a) \mid a \in \mathbb{V}\}=\mathbb{V}^{\{q\}} .
$$

If $\varphi$ is a proposition about sets, then we have in the model the transfer principle: $\varphi((1, A)) \leftrightarrow \varphi(A)$ (i. e., $(1, a) \subset(1, b) \leftrightarrow a \subset b$ etc.).

Now some abstraction of the construction is in order. Put

$$
\mathbb{V}_{Q}:=\mathbb{V}^{Q}:=\{\varphi \mid \varphi: Q \rightarrow \mathbb{V}, \operatorname{dom} \varphi=Q, \operatorname{im} \varphi \subset \mathbb{V}\}
$$

Let $\mathscr{A}=\mathscr{A}(\cdot)$ be a member of $\mathbb{V}_{Q}$ and let $\varphi$ be a formula of set theory. The law of coordinate-wise modeling is obvious:

$$
\varphi(\mathscr{A}) \leftrightarrow(\forall q \in Q) \varphi(\mathscr{A}(q))
$$

With this rule $\mathbb{V}_{Q}$ is clearly a model of set theory. But it seems that such "layer-wise" models brings about nothing new (despite the fact that the model is nonstandard).

Inspect $\mathbb{V}_{Q}$ with more attention. To this end put

$$
\llbracket \varphi(\mathscr{A}) \rrbracket:=\{q \in Q \mid \varphi(\mathscr{A}(q))\} .
$$

We have the truth value of a formula $\varphi$ as the function $\llbracket \cdot \rrbracket$ with values in $\mathscr{P}(Q)$. Clearly, the transfer principle holds:

$$
\text { ( } \varphi \text { is a theorem }) \leftrightarrow \llbracket \varphi \rrbracket=Q .
$$

The following relations are easy:

$$
\begin{gathered}
\llbracket \varphi \wedge \psi \rrbracket=\llbracket \varphi \rrbracket \cap \llbracket \psi \rrbracket, \\
\llbracket \varphi \vee \psi \rrbracket=\llbracket \varphi \rrbracket \cup \llbracket \psi \rrbracket, \llbracket(\forall x) \varphi \rrbracket=\bigwedge_{x} \llbracket \varphi(x) \rrbracket, \\
\llbracket(\exists x) \varphi \rrbracket=\bigvee_{x} \llbracket \varphi(x) \rrbracket,
\end{gathered}
$$

Moreover, we have the maximum principle:

$$
(\exists x) \varphi(x) \rightarrow(\exists x) \llbracket \varphi(x) \rrbracket=Q .
$$


Look at $\mathscr{R}:=\mathbb{R}^{Q}$. Clearly,

$\llbracket \mathscr{R}$ is the field of reals $\rrbracket=Q ;$

i. e., $\mathscr{R}$ models the reals inside $\mathbb{V}_{Q}$.

In other words,

$$
\mathscr{R} \downarrow:=\left\{z \in \mathbb{V}_{Q} \mid \llbracket z \in \mathscr{R} \rrbracket=Q\right\}=\mathbb{R}^{Q},
$$

i. e. The descent of the reals inside $\mathbb{V}_{Q}$ is the space of real functions on $Q$.

We can somehow abstract this construction by replacing $Q$ with the Stone space of some complete Boolean algebra $B$, and $\mathscr{P}(Q)$, with the clopen algebra of $Q$. Strictly speaking, to define the new nonstandard universe $\mathbb{V}^{(B)}$, put

$$
\begin{gathered}
\mathbb{V}_{\alpha}^{(B)}:=\left\{x \mid(\exists \beta \in \alpha) x: \operatorname{dom}(x) \rightarrow B \wedge \operatorname{dom}(x) \subset \mathbb{V}_{\beta}^{(B)}\right\}, \\
\mathbb{V}^{(B)}:=\bigcup_{\alpha \in \text { On }} \mathbb{V}_{\alpha}^{(B)},
\end{gathered}
$$

where $\alpha$ ranges over the class of all ordinals.

The Boolean truth value $\llbracket \varphi \rrbracket$ is defined by recursion on the complexity of $\varphi$ while using the natural interpretation of the logical connectives and quantifies. The truth values of the atomic formulas $x \in y$ and $x=y$ for $x, y \in \mathbb{V}^{(B)}$ are defined by transfinite recursion:

$$
\begin{gathered}
\llbracket x \in y \rrbracket:=\bigvee_{z \in \operatorname{dom}(y)} y(z) \wedge \llbracket z=x \rrbracket ; \\
\llbracket x=y \rrbracket:=\bigwedge_{z \in \operatorname{dom}(x)} x(z) \Rightarrow \llbracket z \in y \rrbracket \wedge \bigwedge_{z \in \operatorname{dom}(y)} y(z) \Rightarrow \llbracket z \in x \rrbracket .
\end{gathered}
$$

(Here $\Rightarrow$ stands for implication in B.), i. e. $a \Rightarrow b:=a^{\perp} \vee b$.)

This we arrive at the Boolean valued universe $\mathbb{V}^{(B)}$ modeling the von Neumann universe $\mathbb{V}$. The reals $\mathscr{R}$ inside the new models has as its descent the universally complete Kantorovich space $\mathscr{R} \downarrow$ whose base in isomorphic to the initial Boolean algebra $B$. This leads to justification of the Kantorovich heuristic principle, and the theory of Boolean valued models turns into one of the powerful tools of vector lattice, a classical section of functional analysis.

The theory of Boolean valued models stems from Dana Scott, Robert Solovay, Petr Vopěnka, and Gaisi Takeuti. These models naturally embrace the nonstandard models by Robinson.

But the importance of infinitesimal methods is so great that nonstandard analysis takes a rather special place in mathematics.

Modeling nonstandard analysis, we pass from the usual von Neumann universe to the "rigged" unverse $\mathbb{V}^{I}$ of the so-called internal sets 
with marked frame points, standard sets, which comprise the copy $\mathbb{V}^{S}$ of $\mathbb{V}$. Further analysis shows that $\mathbb{V}^{I}$ lies in another class, the universe $\mathbb{V}^{E}$ of external sets satisfying Zermelo axioms. Some universe $\mathbb{V}^{C}$ of classical sets is distinguished in $\mathbb{V}^{E}$ which is another realization of the universe of standard sets. Precisely speaking, there is available some *-mapping that identifies $\mathbb{V}^{C}$ and $\mathbb{V}^{S}$ element-wisely. By analogs of transfer principles, $\mathbb{V}^{C}, \mathbb{V}^{S}$, and $\mathbb{V}^{I}$ may be treated as hypostases of the von Neumann universe $\mathbb{V}$. This complicated interaction of nonstandard models is the background of modern reconsideration and enrichment of the ancient infinitesimal methods.

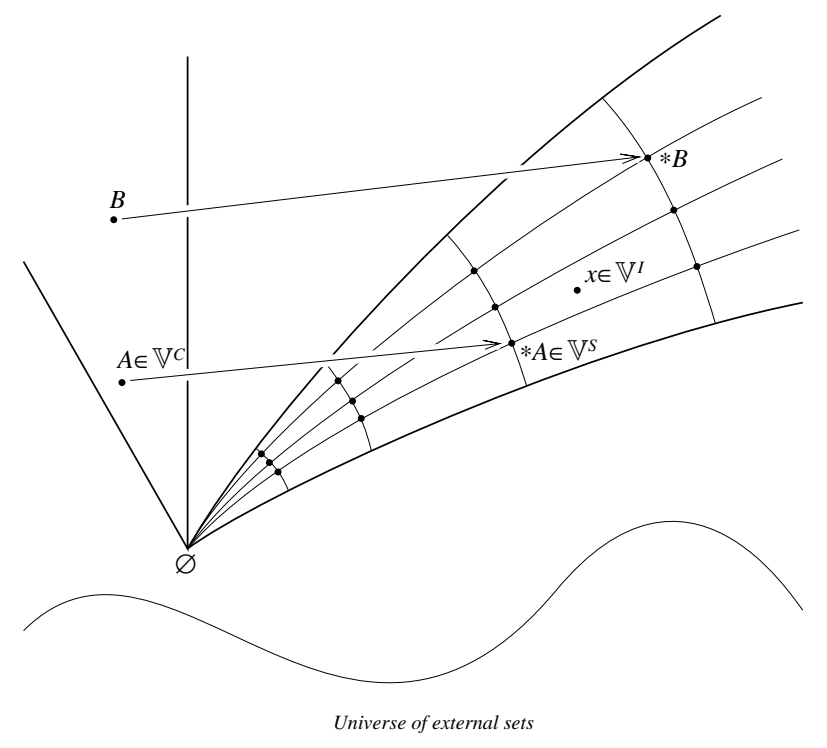

\section{Place of Nonstandard Analysis}

Robinson's nonstandard analysis summarizes the two millennia of the history of views on actual and potential infinities and paves way to the future of the classical calculus, suggesting a new paradigm of foundations which is free from many restrictions of categoricity, prejudice, and bias.

Nonstandard analysis is understood rather broadly today and considered as the branch of mathematics that bases on nonstandard models of set theory. In actually this means that under study are two interacting models simultaneously. Many versions of nonstandard analysis are constructed axiomatically. The most popular are Nelson's Internal Set Theory and Kawai's Nonstandard Set Theory 7 These theories

\footnotetext{
${ }^{7} \mathrm{Cp} \cdot[13]-[17]$.
} 
formalized the ideas that stem from the ancient views of distinctions between actual and potential infinity. The theories are conservative extension of Zermelo-Fraenkel Set Theory, having the same status of justification and rigor in used as for foundations of mathematics. But the new theories provide new expressible opportunities for modelling, analyzing, and solving theoretical and applied problems.

The principal starting point of an axiomatic of nonstandard analysis is the conception that there are two types of origin objects. The elements of one type are available to us either immediately or by a potentially infinite processes in the sense that we can describe such an element directly or prove its unique existence using already available objects. The available elements are called standard and the others are called nonstandard. Nonstandard analysis postulates that each infinite collection of objects has at least one nonstandard element, and every collection of standard elements is itself standard. This implies the transfer principle that asserts the cognizability of a standard mathematical property of any standard collection from the properties of its standards members.

It is worth emphasizing that nonstandard analysis in axiomatic form uses some new primary concept, the property of every element to be or not to be standard. In the "standard" - classical-mathematics of today this property cannot be expressed and so we cannot speak about actual infinitely large or infinitely small elements. Moreover, the formal theory of nonstandard analysis is a conservative extension of the classical set theory. This means that every proposition of classical mathematics which is proved by using nonstandard analysis can be demonstrated without them new nonstandard tools. This leads to the popular misconception that nonstandard analysis can add nothing new. In fact nonstandard analysis is capable of studying the properties of actual infinites and infinitesimals, suggesting new methods of modeling or illuminating the methods of the creators of the calculus like Newton, Leibniz, and Euler who used actual infinities and discriminated between the assignable and nonassignable reals.

All in all, nonstandard analysis opens up some new opportunities that are unavailable in "standard" mathematics. In other words, nonstandard analysis studies the same objects as common mathematics, but it sees in each object The method of nonstandard analysis is sometimes compared with color TV. A black and white TV set can see the same objects as a color TV set, but it cannot discern the variety of colors of the constituents of these elements. This analogy illustrates the fact that the role of nonstandard analysis is much broader that providing extra tools for simplifying the apparatus of standard mathematics 
by the technique of "killing quantifies." Nonstandard analysis reveals the rich inner structure of the classical mathematical objects that are filled with various available and imaginable elements.

To survey the impact of the ideas of nonstandard analysis is as impossible as to survey applications of calculus or probability. Robinson's formalism finds applications in mathematical economics, management, programming, hydrodynamics, optimization, and elsewhere. The formalism of Nelson's Internal Set Theory has expanded and enriched the methodology and the sphere of applications of nonstandard analysis. The new paradigm is connected with reconsideration of the view of the classical continuum. In Nelson's theory infinitesimal reside within the unit segment rather than in ir nonstandard extension as in Robinson's theory. It is impossible to leave unmarked the rehabilitation of the Mises frequency approach which was implemented by Edward Nelson in his conception of "radically elementary probability theory." The external set theories by Toru Kawai and Karel Hrbáček enriched the descriptive and technical possibilities of nonstandard analysis, combining the merits of Robinson's and Nelson's formalisms.

Mathematics must constantly fit itself to the common paradigms of science. Nonstandard analysis crowns the old-fashioned ideas of the ancient atomism in much the same way as the Lobachevsky geometry terminated the dogmatical period of the development of Euclidean geometry. Robinson suggested a new outlook on the history of mathematical concepts that underlie the foundations of analysis. His views and approaches proliferate rapidly these days 8

The twentieth century is marked with liberation of humankind from dogmatism and uniformity. Filled with the inflammable mixture of genius and villainy of the population of Homo Sapiens, the twentieth century will remain in history the age of liberation of humankind from fascism, categoricity, absolutism, and domination rather than the age of hatred and cannibalism. Nonstandard analysis is a produce and source of freedom.

Humankind will never waste out its intellectual treasures. Thus there is no doubt that the Gödel forecast of the future of nonstandard analysis will turn out prophetic, and some version of nonstandard analysis will take place of the classical differential and integral calculus of today. Differentiation as search of trends and integration as prediction of future from trends are immortal technologies of mind. New technologies are awaiting humankind which will use the whole of mathematics

\footnotetext{
${ }^{8}$ Cp. [18].
} 
in portions incomprehensible today. This will be the analysis of the future Gödel had written about.

\section{REFERENCES}

[1] Mostow G., "Abraham Robinson." Israel Math. J., 25:1-2, 5-14 (1976).

[2] Macintyre A., "Abhaham Robinson, 1918-1974." Bull. Amer. Math. Soc., 83:4, 646-666 (1977).

[3] Dauben J. W., Abraham Robinson: The Creation of Nonstandard Analysis: A Personal and Mathematical Oddyssey. Princeton University Press. Princeton, 1995.

[4] Halmos P., I Want to Be a Mathematician. An Automathography. SpringerVerlag, New York etc., 1985.

[5] Luxemburg W. A. J. (ed.), Applications of Model Theory to Algebra, Analysis and Probability. Holt, Rinehart, and Winston; New York, 1969.

[6] Luxemburg W. A. J., "A nonstandard approach to Fourier analysis," in: Contributions to Nonstandard Analysis, North-Holland, Amsterdam, 1972, pp. 1639.

[7] Robinson A., Selected Papers, Vol. 2. Nonstandard Analysis and Philosophy. Yale University Press, New Haven and London, 1979.

[8] Robinson A., Introduction to the Theory of Models and to the Metamathematics of Algebra. North-Holland, Amsterdam, 1963.

[9] Robinson A., "The metaphysics of the calculus," in: Problems in the Philosophy of Mathematics, North-Holland, Amsterdam, 1967, 1, pp. 28-46.

[10] Luxemburg W. A. J. and Robinson A. (eds.), Contribution to Non-Standard Analysis. North-Holland, Amsterdam, 1972.

[11] Robinson A., Non-Standard Analysis. Princeton Univ. Press, Princeton,1996.

[12] Gödel K., Collected Works: Correspondence. Vols. 4 and 5. Oxford University Press, Oxford, 2003.

[13] Nelson E., "Internal set theory. A new approach to nonstandard analysis," Bull. Amer. Math. Soc., 83, No. 6, 1165-1198 (1977).

[14] Nelson E., Radically Elementary Probability Theory. Princeton University Press, Princeton, 1987.

[15] Nelson E., "The syntax of nonstandard analysis," Ann. Pure Appl. Logic, 38:2, 123-134 (1988).

[16] Kanovei V. and Reeken M., Nonstandard Analysis, Axiomatically. SpringerVerlag, Berlin, 2004.

[17] Gordon E. I., Kusraev A. G., and Kutateladze S. S., Infinitesimal Analysis: Selected Topics. Nauka, Moscow, 2011.

[18] Bair J., Błaszczyk P., Ely R., Henry V., Kanovei V., Katz K., Katz M., Kutateladze S., McGaffey T., Schaps D., Sherry D., and Shnider S., "Is mathematical history written by the victors?" Notices of the AMS, 60:7, 886-904 (2013).

Sobolev Institute of Mathematics

NovosiBIRsk 630090, RUSSIA

E-mail address: $\quad$ sskut@math.nsc.ru 